\title{
Relations among several nuclear and electronic density functional reactivity indexes
}

Miquel Torrent-Sucarrat, Josep M. Luis, and Miquel Duran

Institut de Quimica Computacional and Departament de Química, Universitat de Girona, E-17071 Girona, Catalonia, Spain

Alejandro Toro-Labbé

Facultad de Química, Pontificia Universidad Católica de Chile, Casilla 306, Correo 22, Santiago, Chile

Miquel Solàa)

Institut de Quimica Computacional and Departament de Química, Universitat de Girona, E-17071 Girona, Catalonia, Spain

(Received 7 July 2003; accepted 13 August 2003)

\begin{abstract}
An expansion of the energy functional in terms of the total number of electrons and the normal coordinates within the canonical ensemble is presented. A comparison of this expansion with the expansion of the energy in terms of the total number of electrons and the external potential leads to new relations among common density functional reactivity descriptors. The formulas obtained provide explicit links between important quantities related to the chemical reactivity of a system. In particular, the relation between the nuclear and the electronic Fukui functions is recovered. The connection between the derivatives of the electronic energy and the nuclear repulsion energy with respect to the external potential offers a proof for the "Quantum Chemical le Chatelier Principle." Finally, the nuclear linear response function is defined and the relation of this function with the electronic linear response function is given. (C) 2003 American Institute of Physics.
\end{abstract}

[DOI: $10.1063 / 1.1615763$ ]

\section{INTRODUCTION}

According to Density Functional Theory (DFT), ${ }^{1}$ the electronic energy of a many-electron system is given by

$$
E[\rho]=F[\rho]+\int \nu(\vec{r}) \rho(\vec{r}) d \vec{r},
$$

where $F[\rho]$ is the universal Hohenberg-Kohn functional defined as the sum of the electronic kinetic energy functional, $T[\rho]$, and the electron-electron interaction energy functional, $V_{e e}[\rho]$, and $\nu(\vec{r})$ is the external potential that for an isolated molecule is just the potential due to atomic nuclei, but in general it may also include contributions from external fields or neighboring molecules. Minimization of the electronic energy with respect to the electron density with the constraint of constant total number of electrons, $N$, leads to an Euler equation of the form

$$
\mu=\nu(\vec{r})+\frac{\delta F[\rho]}{\delta \rho(\vec{r})} .
$$

The solution to this equation yields the ground state electron density, from which one can determine the ground state energy.

Since the number of electrons and the external potential completely determine the Hamiltonian of the system, one can write the electronic energy of the system as a functional of $N$ and $\nu(\vec{r})$ (i.e., $E=E[N, \nu]$ ). On the other hand, Eq. (1) establishes that $E$ is a functional of $\rho(\vec{r})$ and $\nu(\vec{r})$ (i.e., $E$

${ }^{\text {a)} C o r r e s p o n d i n g ~ a u t h o r . ~ E l e c t r o n i c ~ m a i l: ~ m i q u e l . s o l a @ u d g . e s ~}$
$=E[\rho, \nu])$. Differential expansion of the electronic energy, $E$, for these two energy functionals allows us to determine the physical significance of the Lagrange multiplier, $\mu$, which is found to be the electronic chemical potential of the system, that is,

$$
\mu=\left(\frac{\partial E}{\partial N}\right)_{\nu}
$$

and to find that the density is the functional first derivative of the energy with respect to the external potential at a constant total number of electrons, i.e.,

$$
\rho(\vec{r})=\left(\frac{\delta E}{\delta \nu(\vec{r})}\right)_{N} .
$$

Second-order derivatives of the energy with respect to $N$ and $\nu(\vec{r})$ also have clear physical meanings. ${ }^{2}$ Thus, the second derivative with respect to the number of electrons at a fixed external potential $\nu(\vec{r})$ is the hardness: ${ }^{3}$

$$
\eta=\left(\frac{\partial^{2} E}{\partial N^{2}}\right)_{\nu}
$$

while the second derivative with respect to the external potential $\nu(\vec{r})$ at a fixed number of electrons is the linear response function, ${ }^{4}$ 


$$
\chi\left(\vec{r}, \vec{r}^{\prime}\right)=\left(\frac{\delta^{2} E}{\delta \nu(\vec{r}) \delta \nu\left(\vec{r}^{\prime}\right)}\right)_{N}=\left(\frac{\delta \rho(\vec{r})}{\delta \nu\left(\vec{r}^{\prime}\right)}\right)_{N},
$$

which measures the variation of the electron density at point $r$ when the external potential of the system is locally perturbed at $r^{\prime}$. Finally, the Fukui function, first defined by Parr and Yang, ${ }^{5}$ is the second derivative with respect to external potential $\nu(\vec{r})$ and the number of electrons,

$$
f(\vec{r})=\left(\frac{\delta^{2} E}{\partial N \delta \nu(\vec{r})}\right)=\left(\frac{\partial \rho(\vec{r})}{\partial N}\right)_{\nu}=\left(\frac{\delta \mu}{\delta \nu(\vec{r})}\right)_{N} .
$$

The Fukui function describes the local changes in the electron density of the system, due to the perturbation in the global number of electrons, so it reflects the character of a molecule to accept (donate) electrons from (to) an another system. For a molecular or atomic system, the density is a discrete function of the number of electrons. Because of that, Parr and Yang ${ }^{5}$ associated different physical meanings to the left, right, and central derivative of the density with respect to $N$, corresponding to a reactivity index for a nucleophilic $f^{+}(\vec{r})$, electrophilic $f^{-}(\vec{r})$, and radical $f^{0}(\vec{r})$ attacks, respectively. By applying a finite difference approximation to Eq. (7), these three approximate Fukui functions can be written as

$$
\begin{aligned}
& f^{+}(\vec{r})=\rho_{N+1}(\vec{r})-\rho_{N}(\vec{r}), \\
& f^{-}(\vec{r})=\rho_{N}(\vec{r})-\rho_{N-1}(\vec{r}),
\end{aligned}
$$

and

$$
f^{0}(\vec{r})=\frac{1}{2}\left[\rho_{N+1}(\vec{r})-\rho_{N-1}(\vec{r})\right],
$$

where $\rho_{N+1}(\vec{r}), \rho_{N}(\vec{r})$, and $\rho_{N-1}(\vec{r})$ are the electronic densities of the system with $N+1, N$, and $N-1$ electrons, respectively.

Upon the course of a chemical reaction, molecules adjust their number of electrons and their external potential. This implies changes in both the electronic structure, characterized by the electron density $\rho(\vec{r})$, and the geometric structure defined by the position vectors $\left\{R_{\alpha}\right\}$ of the nuclei. To describe the electronic changes, one can use the Fukui function, the hardness, and the linear response function. These are electronic indexes that measure the electron density and electronic chemical potential responses to a change in $N$ or $\nu(\vec{r})$. A Kohn-Sham formulation of the chemical electronic responses has been provided by Senet. ${ }^{6}$ To describe the geometrical changes, a set of nuclear reactivity indexes that characterize the response of the nuclei due to changes in $N$ or $\nu(\vec{r})$ of the system have been recently defined. ${ }^{7,8}$ In particular, the nuclear Fukui function (NFF) has been defined by Cohen and coworkers ${ }^{7}$ as the electronic force $F$ acting on the nuclei due to the perturbation in $N$ at a constant $\nu(\vec{r})$ :

$$
\phi_{\alpha}=\left(\frac{\partial \vec{F}_{\alpha}}{\partial N}\right)_{\nu} .
$$

In analogy with the electronic Fukui function, it is possible to define three NFFs corresponding to nucleophilic, electrophilic, and radical attacks as ${ }^{9}$

$$
\begin{gathered}
\phi_{\alpha}^{+}=\left(\frac{\partial \vec{F}_{\alpha}}{\partial N}\right)_{\nu}^{+}=\vec{F}_{\alpha}(N+1)-\vec{F}_{\alpha}(N)=-\vec{\nabla}_{\alpha} E(N+1), \\
\phi_{\alpha}^{-}=\left(\frac{\partial \vec{F}_{\alpha}}{\partial N}\right)_{\nu}^{-}=\vec{F}_{\alpha}(N)-\vec{F}_{\alpha}(N-1)=\vec{\nabla}_{\alpha} E(N-1), \\
\phi_{\alpha}^{0}=\left(\frac{\partial \vec{F}_{\alpha}}{\partial N}\right)_{\nu}^{0}=\frac{1}{2}\left(\vec{F}_{\alpha}(N+1)-\vec{F}_{\alpha}(N-1)\right) \\
=\frac{1}{2}\left(\vec{\nabla}_{\alpha} E(N-1)-\vec{\nabla}_{\alpha} E(N+1)\right) .
\end{gathered}
$$

The last equality in Eqs. (12) and (13) is only valid when the $N$ electron system of reference is at its equilibrium geometry $\left[\vec{F}_{\alpha}(N)=0\right]$.

Baekelandt $^{8}$ via a Maxwell relation has shown that this NFF can be interpreted as the conformational contribution to the change in the electronic chemical potential:

$$
\phi_{\alpha}=\left(\frac{\partial \vec{F}_{\alpha}}{\partial N}\right)_{\nu}=-\left(\frac{\partial^{2} E}{\partial N \delta \vec{R}_{\alpha}}\right)=-\left(\frac{\delta \mu}{\delta \vec{R}_{\alpha}}\right)_{N} .
$$

From Eq. (15), three definitions of the NFF corresponding to the left, right, and central derivatives are also possible: ${ }^{10}$

$$
\begin{gathered}
\phi_{\alpha}^{+}=-\left(\frac{\partial^{2} E}{\partial N \delta \vec{R}_{\alpha}}\right)^{+} \cong\left(\frac{\delta A}{\delta \vec{R}_{\alpha}}\right)_{N} \cong-\left(\frac{\delta \varepsilon_{\mathrm{LUMO}}}{\delta \vec{R}_{\alpha}}\right)_{N}, \\
\phi_{\alpha}^{-}=-\left(\frac{\partial^{2} E}{\partial N \delta \vec{R}_{\alpha}}\right)^{-} \cong\left(\frac{\delta I}{\delta \vec{R}_{\alpha}}\right)_{N} \cong-\left(\frac{\delta \varepsilon_{\mathrm{HOMO}}}{\delta \vec{R}_{\alpha}}\right)_{N},
\end{gathered}
$$

and

$$
\phi_{\alpha}^{0}=-\left(\frac{\partial^{2} E}{\partial N \delta \vec{R}_{\alpha}}\right)^{0} \cong\left(\frac{\delta \mu_{2}}{\delta \vec{R}_{\alpha}}\right)_{N} \cong\left(\frac{\delta \mu_{1}}{\delta \vec{R}_{\alpha}}\right)_{N},
$$

where the Koopmans' theorem ${ }^{11}$ has been used in Eqs. (16) and (17), and $\mu_{2}$ and $\mu_{1}$ in Eq. (18) refer to the following operational approximations for the electronic chemical potential:

$$
\begin{aligned}
& \mu_{2}=-\frac{1}{2}(I+A), \\
& \mu_{1}=\frac{1}{2}\left(\varepsilon_{L}+\varepsilon_{H}\right) .
\end{aligned}
$$

It is remarkable the similarity between Eqs. (7) and (15). Geerlings and coworkers have computed NFFs for diatomic molecules using Eqs. (12) and (13), as well as from numerical calculations of the negative derivative of the chemical potential with respect to the atomic coordinates [Eqs. (16) and (17) $]^{9}$ and with an approximate analytical method ${ }^{10}$ developed in analogy with Komorowski and Balawender's ${ }^{12}$ coupled Hartree-Fock approach to the electronic Fukui function. They have found that analytical and numerical results show a high correlation. Finally, it is worth mentioning that the relationship between the NFFs and the Berlin's binding 
function $^{13}$ has been recently discussed ${ }^{8,9,14}$ and new definitions of nuclear/geometric reactivity indexes have been also put forward. ${ }^{15-17}$ These studies ${ }^{8-12,14-17}$ have reinforced the idea that a complete representation of the total chemical response to a given perturbation must involve the analysis of both electronic and nuclear reactivity descriptors.

Because of the coupling between the electronic and nuclear responses to external perturbations, one can expect that relationships between nuclear and reactivity indexes exist. In the present paper we apply the general functional expansion scheme to expand the potential energy, $U$, and the total electronic energy, $E$, of the systems in terms of the total number of electrons, $N$, and normal coordinates displacements, $\left\{Q_{k}\right\}$, to derive relationships between electronic and nuclear indexes that connect the electronic and nuclear responses in front of a given perturbation. The energy expansion is carried out within the canonical ensemble, for which the natural variables are the global variable $N$ and the local variable $\nu(\vec{r})$.

\section{ENERGY REPRESENTATIONS}

In the Born-Oppenheimer approximation, the Potential Energy Surface (PES) of a system can be divided into an electronic $(E)$ and nuclear repulsion $\left(V_{n n}\right)$ energy terms as

$$
U[\rho, \nu]=E[\rho, \nu]+V_{n n}[\nu] .
$$

As stated for the electronic energy, one can also write the PES of the system as a functional of $N$ and $\nu(\vec{r})$, i.e., $U$ $=U[N, \nu]$ :

$$
U[N, \nu]=E[N, \nu]+V_{n n}[\nu] .
$$

A Taylor series functional expansion of the PES around a reference external potential $\nu_{o}(\vec{r})$ and total number of electrons $N$, retaining terms up to second order, acquires the form (note that the nuclear repulsion term does not depend on $N$ )

$$
\begin{aligned}
\Delta U= & \left(\frac{\partial E}{\partial N}\right)_{\nu} \Delta N+\int_{\nu}\left(\frac{\delta E}{\delta \nu(\vec{r})}\right)_{N} \Delta \nu(\vec{r}) d \vec{r} \\
& +\int\left(\frac{\delta V_{n n}}{\delta \nu(\vec{r})}\right)_{N} \Delta \nu(\vec{r}) d \vec{r}+\frac{1}{2}\left(\frac{\partial^{2} E}{\partial N^{2}}\right)_{\nu} \Delta N^{2} \\
& +\frac{1}{2} \iint\left(\frac{\delta^{2} E}{\delta \nu(\vec{r}) \delta \nu\left(\vec{r}^{\prime}\right)}\right)_{N} \Delta \nu(\vec{r}) \Delta \nu\left(\vec{r}^{\prime}\right) d \vec{r} d \vec{r}^{\prime} \\
& +\frac{1}{2} \iint\left(\frac{\delta^{2} V_{n n}}{\delta \nu(\vec{r}) \delta \nu\left(\vec{r}^{\prime}\right)}\right)_{N} \Delta \nu(\vec{r}) \Delta \nu\left(\vec{r}^{\prime}\right) d \vec{r} d \vec{r}^{\prime} \\
& +\int\left(\frac{\delta^{2} E}{\delta \nu(\vec{r}) \partial N}\right) \Delta N \Delta \nu(\vec{r}) d \vec{r}
\end{aligned}
$$

Equation (23) is the same used by Ayers and Parr to explore the effect that changing the external potential has on chemical reactivity. ${ }^{18}$ Now, substituting Eqs. (3) to (7) into Eq. (23) one finds that

$$
\begin{aligned}
\Delta U= & \mu \Delta N+\int \rho(\vec{r}) \Delta \nu(\vec{r}) d \vec{r}+\int\left(\frac{\delta V_{n n}}{\delta \nu(\vec{r})}\right)_{N} \Delta \nu(\vec{r}) d \vec{r} \\
& +\frac{1}{2} \eta \Delta N^{2}+\frac{1}{2} \iint \chi\left(\vec{r}, \vec{r}^{\prime}\right) \Delta \nu(\vec{r}) \Delta \nu\left(\vec{r}^{\prime}\right) d \vec{r} d \vec{r}^{\prime} \\
& +\frac{1}{2} \iint\left(\frac{\delta^{2} V_{n n}}{\delta \nu(\vec{r}) \delta \nu\left(\vec{r}^{\prime}\right)}\right)_{N} \Delta \nu(\vec{r}) \Delta \nu\left(\vec{r}^{\prime}\right) d \vec{r} d \vec{r}^{\prime} \\
& +\Delta N \int f(\vec{r}) \Delta \nu(\vec{r}) d \vec{r} .
\end{aligned}
$$

Let us now consider a molecule in an isotropic and homogeneous environment. In this case, the external potential depends only upon the relative nuclear positions. These relative positions can be specified using a set of $3 N-6$ (or $3 N-5$ for linear molecules) coordinates, the most natural choice being the use of the normal coordinates, $\left\{Q_{k}\right\}$. Given that the external potential for an isolated molecule can be defined from a set of $\left\{Q_{k}\right\}$ coordinates, one can write the energy of this system as a functional of $N$ and $\left\{Q_{k}\right\}$, i.e., $U$ $=U\left[N,\left\{Q_{k}\right\}\right]$. In this functional form, a Taylor series expansion of the total energy up to second order yields

$$
\begin{aligned}
\Delta U= & \left(\frac{\partial E}{\partial N}\right)_{\nu\left\{Q_{k}\right\}} \Delta N+\sum_{k=1}^{3 N-6}\left(\frac{\partial U}{\partial Q_{k}}\right)_{N} \Delta Q_{k} \\
& +\frac{1}{2}\left(\frac{\partial^{2} E}{\partial N^{2}}\right)_{\nu\left\{Q_{k}\right\}} \Delta N^{2}+\sum_{k=1}^{3 N-6}\left(\frac{\partial^{2} U}{\partial Q_{k}^{2}}\right)_{N} \Delta Q_{k}^{2} \\
& +\sum_{k=1}^{3 N-6}\left(\frac{\partial^{2} E}{\partial N \partial Q_{k}}\right) \Delta Q_{k} \Delta N,
\end{aligned}
$$

$\Delta Q_{k}$ being the displacement of the normal mode $k$ from the equilibrium position. It is worth noting that since the external potential can be written as a function of the normal coordinates, it is possible to transform the derivatives with respect to the external potential to derivatives with respect to normal coordinates by using the chain rule. In this way, it is also possible to derive Eq. (25) from Eq. (23).

If we take as the reference state a molecule in its equilibrium geometry, then the term

$$
\sum_{k=1}^{3 N-6}\left(\frac{\partial U}{\partial Q_{k}}\right){ }_{N} \Delta Q_{k},
$$

in Eq. (25) and the term

$$
\begin{aligned}
& \int\left(\frac{\delta U}{\delta \nu(\vec{r})}\right)_{N} \Delta \nu(\vec{r}) d \vec{r} \\
& \quad=\int \rho(\vec{r}) \Delta \nu(\vec{r}) d \vec{r}+\int\left(\frac{\delta V_{n n}}{\delta \nu(\vec{r})}\right)_{N} \Delta \nu(\vec{r}) d \vec{r},
\end{aligned}
$$

in Eq. (24) are zero. We note in passing that Eq. (27) leads to the following interesting relationship that holds for an isolated molecule in its equilibrium geometry:

$$
\left(\frac{\delta E}{\delta \nu(\vec{r})}\right)_{N}=\rho(\vec{r})=-\left(\frac{\delta V_{n n}}{\delta \nu(\vec{r})}\right)_{N} .
$$


This equation is the result of the fact that, in the equilibrium geometry, the electronic energy change due to a perturbation in $\nu(\vec{r})$ equals the negative of nuclear repulsion energy change, providing a relation between electronic and nuclear reactivity descriptors. Further, it is a nice proof to the firstorder and within the framework of conceptual $\mathrm{DFT}^{19}$ of the "Quantum Chemical le Chatelier Principle" formulated by Mezey some years ago. ${ }^{20}$ This principle states that the electronic energy change induced by an external perturbation is compensated by a modification of the nuclear repulsion energy by a similar amount (in absolute value), in such a way that the value of the total energy (electronic + nuclear) is approximately conserved.

Now, from Eq. (25), using Eqs. (3) and (5) and taking into account that

$$
\left(\frac{\partial^{2} E}{\partial N \partial Q_{k}}\right)=-\left(\frac{\partial F_{k}}{\partial N}\right)_{\nu\left\{Q_{k}\right\}}=-\phi_{k}
$$

and

$$
\left(\frac{\partial^{2} U}{\partial Q_{k}^{2}}\right)_{N}=\lambda_{k},
$$

one gets

$$
\begin{aligned}
\Delta U= & \mu \Delta N+\frac{1}{2} \eta \Delta N^{2}+\frac{1}{2} \sum_{k=1}^{3 N-6} \lambda_{k} \Delta Q_{k}^{2} \\
& -\Delta N \sum_{k=1}^{3 N-6} \phi_{k} \Delta Q_{k} .
\end{aligned}
$$

Equation (31) holds for a molecule in its equilibrium geometry. In Eqs. (29) and (31), $\phi_{k}$ are the NFFs in terms of the normal coordinates and, in Eqs. (30) and (31), $\lambda_{k}$ are the force constants of each normal mode.

A comparison of Eqs. (24) and (31) leads to Eqs. (32) and (34). Equation (32) is obtained, considering the independent terms with respect to $\Delta N$ :

$$
\begin{aligned}
\frac{1}{2} \iint \mathrm{X}\left(\vec{r}, \vec{r}^{\prime}\right) \Delta \nu(\vec{r}) \Delta \nu\left(\vec{r}^{\prime}\right) d \vec{r} d \vec{r}^{\prime} \\
\quad+\frac{1}{2} \iint \chi\left(\vec{r}, \vec{r}^{\prime}\right) \Delta \nu(\vec{r}) \Delta \nu\left(\vec{r}^{\prime}\right) d \vec{r} d \vec{r}^{\prime} \\
\quad=\frac{1}{2} \sum_{k=1}^{3 N-6} \lambda_{k} \Delta Q_{k}^{2},
\end{aligned}
$$

and defining the nuclear linear response function as

$$
\mathrm{X}\left(\vec{r}, \vec{r}^{\prime}\right)=\left(\frac{\delta^{2} V_{n n}}{\delta \nu(\vec{r}) \delta \nu\left(\vec{r}^{\prime}\right)}\right)_{N},
$$

while Eq. (34) appears matching the terms that depend on $\Delta N$ :

$$
\int f(\vec{r}) \Delta \nu(\vec{r}) d \vec{r}=-\sum_{k=1}^{3 N-6} \phi_{k} \Delta Q_{k} .
$$

Equation (32) affords a relation for the nuclear and electronic linear response functions. For small $\left\{Q_{k}\right\}$ displacements, the term (1/2) $\sum_{k=1}^{3 N-6} \lambda_{k} \Delta Q_{k}^{2}$ in Eq. (32) gives the total change in the potential energy due to nuclear rearrangement. Equation (32) shows that this change can be decomposed in two parts: the first term, $(1 / 2) \iint \mathrm{X}\left(\vec{r}, \vec{r}^{\prime}\right) \Delta \nu(\vec{r}) \Delta \nu\left(\vec{r}^{\prime}\right) d \vec{r} d \vec{r}^{\prime}$, represents the change of the nuclear potential energy due to the distortion while the remaining term is the change in electronic energy.

Equations (32) and (34) are the result of the Taylor series expansion of the PES functionals $U=U[N, \nu]$ and $U$ $=U\left[N,\left\{Q_{k}\right\}\right]$. Similarly, one can expand the functionals of the electronic energy $E=E[N, \nu]$ and $E=E\left[N,\left\{Q_{k}\right\}\right]$. Following the same procedure one recovers Eq. (34) and also a modified version of Eq. (32) that reads as

$$
\begin{aligned}
& \frac{1}{2} \int \int \chi\left(\vec{r}, \vec{r}^{\prime}\right) \Delta \nu(\vec{r}) \Delta \nu\left(\vec{r}^{\prime}\right) d \vec{r} d \vec{r}^{\prime} \\
& \quad=\frac{1}{2} \sum_{k=1}^{3 N-6} \sum_{l=1}^{3 N-6}\left(\frac{\partial^{2} E}{\partial Q_{k} \partial Q_{l}}\right)_{N} \Delta Q_{k} \Delta Q_{l} .
\end{aligned}
$$

Considering now the relation between the electronic energy and the PES given by Eq. (22), one obtains

$$
\begin{aligned}
& \frac{1}{2} \iint \chi\left(\vec{r}, \vec{r}^{\prime}\right) \Delta \nu(\vec{r}) \Delta \nu\left(\vec{r}^{\prime}\right) d \vec{r} d \vec{r}^{\prime} \\
&= \frac{1}{2} \sum_{k=1}^{3 N-6} \lambda_{k} \Delta Q_{k}^{2}-\frac{1}{2} \sum_{k=1}^{3 N-6} \sum_{l=1}^{3 N-6}\left(\frac{\partial^{2} V_{n n}}{\partial Q_{k} \partial Q_{l}}\right)_{N} \\
& \times \Delta Q_{k} \Delta Q_{l} .
\end{aligned}
$$

This equation provides a way to compute the $(1 / 2) \iint \chi\left(\vec{r}, \vec{r}^{\prime}\right) \Delta \nu(\vec{r}) \Delta \nu\left(\vec{r}^{\prime}\right) d \vec{r} d \vec{r}^{\prime}$ integral from the evaluation of the other two terms in Eq. (36). Remarkably, using Eqs. (36) and (32), it is possible to compute numerically all terms present in Eq. (24). By means of Eq. (24), one could carry out an energy decomposition analysis of the interaction between two given systems. More research on the value of such an energy decomposition analysis is underway in our laboratory.

Finally, from Eqs. (32) and (36) one obtains Eq. (37):

$$
\begin{aligned}
\frac{1}{2} \iint X\left(\vec{r}, \vec{r}^{\prime}\right) \Delta \nu(\vec{r}) \Delta \nu\left(\vec{r}^{\prime}\right) d \vec{r} d \vec{r}^{\prime} \\
=\frac{1}{2} \sum_{k=1}^{3 N-6} \sum_{l=1}^{3 N-6}\left(\frac{\partial^{2} V_{n n}}{\partial Q_{k} \partial Q_{l}}\right)_{N} \Delta Q_{k} \Delta Q_{l},
\end{aligned}
$$

which provides a means to numerically compute the $(1 / 2) \iint X\left(\vec{r}, \vec{r}^{\prime}\right) \Delta \nu(\vec{r}) \Delta \nu\left(\vec{r}^{\prime}\right) d \vec{r} d \vec{r}^{\prime}$ integral.

Coming back to Eq. (34), this equation represents a basic expression that connects the electronic and nuclear Fukui functions. It is a quantitative expression for the coupling of nuclear and electronic responses to perturbations. It was first found by Baekeland ${ }^{8}$ in differential form and in a different way, starting from the definition of the NFF and using the chain rule to express the derivative of the electronic chemical potential with respect to the external potential. According to this author the term $-\sum_{k=1}^{3 N-6} \phi_{k} \Delta Q_{k}$ corresponds to the nuclear-dependent part of the change in the electronic chemical potential. Interestingly, we have recovered this important 
TABLE I. The $\phi_{\alpha}^{+}, \phi_{\alpha}^{-}$, and $\phi_{\alpha}^{0}$ nuclear Fukui functions for 13 diatomic calculated using the different approximations represented by Eqs. (12) to (14) and (16) to (18). All values are given in atomic units.

\begin{tabular}{|c|c|c|c|c|c|c|c|c|c|}
\hline \multirow[b]{2}{*}{ Molecule } & \multicolumn{3}{|c|}{$\phi_{\alpha}^{+}$} & \multicolumn{3}{|c|}{$\phi_{\alpha}^{-}$} & \multicolumn{3}{|c|}{$\phi_{\alpha}^{0}$} \\
\hline & LUMO $^{\mathrm{a}}$ & $A^{\mathrm{b}}$ & $\left(\partial \vec{F}_{\alpha} / \partial N\right)_{\nu}^{+\mathrm{c}}$ & $\mathrm{HOMO}^{\mathrm{a}}$ & $\mathrm{I}^{\mathrm{b}}$ & $\left(\partial \vec{F}_{\alpha} / \partial N\right)_{\nu}^{-\mathrm{c}}$ & $\mu_{1}^{\mathrm{d}}$ & $\mu_{2}{ }^{\mathrm{e}}$ & $\left(\partial \vec{F}_{\alpha} / \partial N\right)_{\nu}^{0 \mathrm{c}}$ \\
\hline $\mathrm{H}_{2}$ & -0.004656 & 0.005600 & 0.005600 & -0.164816 & -0.152412 & -0.152412 & -0.084725 & -0.073406 & -0.073406 \\
\hline $\mathrm{LiH}$ & 0.008700 & 0.009788 & 0.009789 & -0.028850 & -0.027875 & -0.027878 & -0.010075 & -0.009045 & -0.009045 \\
\hline $\mathrm{BH}$ & 0.002550 & 0.007344 & 0.007344 & 0.014231 & 0.015300 & 0.015296 & 0.008396 & 0.011320 & 0.011320 \\
\hline $\mathrm{NH}$ & -0.006150 & 0.004069 & 0.004067 & -0.006225 & -0.027150 & -0.027155 & -0.006187 & -0.011544 & -0.011544 \\
\hline $\mathrm{HF}$ & 0.011669 & 0.013050 & 0.013045 & -0.055213 & -0.085750 & -0.085735 & -0.021772 & -0.036345 & -0.036345 \\
\hline $\mathrm{HCl}$ & 0.006694 & 0.008000 & 0.007973 & -0.013766 & -0.023050 & -0.023055 & -0.003537 & -0.007541 & -0.007541 \\
\hline $\mathrm{N}_{2}$ & 0.003209 & 0.003281 & 0.003280 & -0.239375 & -0.057675 & -0.057678 & -0.118075 & -0.027199 & -0.027199 \\
\hline $\mathrm{F}_{2}$ & 0.104200 & 0.475250 & 0.475245 & 0.127350 & 0.135722 & 0.135722 & 0.115750 & 0.305483 & 0.305483 \\
\hline $\mathrm{CO}$ & -0.009500 & 0.016750 & 0.016738 & 0.067800 & 0.044200 & 0.044197 & 0.029153 & 0.030468 & 0.030468 \\
\hline NF & 0.114562 & 0.132250 & 0.132248 & 0.164812 & 0.146031 & 0.146037 & 0.139694 & 0.139142 & 0.139143 \\
\hline $\mathrm{BF}$ & 0.009131 & 0.011438 & 0.011435 & 0.067762 & 0.068962 & 0.068962 & 0.038447 & 0.040198 & 0.040198 \\
\hline $\mathrm{NO}^{+}$ & 0.302338 & 0.326063 & 0.326065 & -0.039825 & -0.136456 & -0.136455 & 0.131250 & 0.094805 & 0.094805 \\
\hline $\mathrm{LiF}$ & 0.015687 & 0.016000 & 0.016012 & -0.058025 & -0.058938 & -0.058936 & -0.021169 & -0.021462 & -0.021462 \\
\hline
\end{tabular}

${ }^{\mathrm{a}}$ Calculated by evaluating $\phi_{\alpha}^{+} \cong-\left(\delta \varepsilon_{\mathrm{LUMO}} / \delta \vec{R}_{\alpha}\right)_{N}$ or $\phi_{\alpha}^{-} \cong-\left(\delta \varepsilon_{\mathrm{HOMO}} / \delta \vec{R}_{\alpha}\right)_{N}$.

${ }^{\mathrm{b} C a l c u l a t e d}$ by evaluating $\phi_{\alpha}^{+} \cong\left(\delta A / \delta \vec{R}_{\alpha}\right)_{N}$ or $\phi_{\alpha}^{-} \cong\left(\delta I / \delta \vec{R}_{\alpha}\right)_{N}$.

${ }^{\mathrm{c}}$ Calculated using Eqs. (12) to (14).

${ }^{\mathrm{d}}$ Calculated using Eqs. (18) and (20).

${ }^{\mathrm{e}}$ Calculated using Eqs. (18) and (19).

connection between electronic and nuclear Fukui functions through a Taylor functional expansion of the energy as a functional of $N$ and $\left\{Q_{k}\right\}$.

\section{CALCULATION OF THE TERMS INVOLVED IN THE RELATIONS BETWEEN NUCLEAR AND ELECTRONIC REACTIVITY INDEXES}

Throughout this paper we have assumed that all Taylor series converge. Moreover, we expect that if the external potential of the final system resembles that of the reference state sufficiently closely, low-order truncations of these functional Taylor series will be accurate. Our aim in this section is first to check this hypothesis by carrying out calculations of the two terms of Eq. (34). Second, the calculation of the $(1 / 2) \iint \chi\left(\vec{r}, \vec{r}^{\prime}\right) \Delta \nu(\vec{r}) \Delta \nu\left(\vec{r}^{\prime}\right) d \vec{r} d \vec{r}^{\prime}$ and $(1 / 2) \iint \mathrm{X}\left(\vec{r}, \vec{r}^{\prime}\right) \Delta \nu(\vec{r}) \Delta \nu\left(\vec{r}^{\prime}\right) d \vec{r} d \vec{r}^{\prime}$ integrals will be performed for a series of diatomic molecules.

All calculations have been carried out with the GAUSSIAN 98 package $^{21}$ at the Hartree-Fock (HF) ${ }^{22}$ level using the aug-cc-pVQZ basis set, ${ }^{23}$ except for the lithium atom, for which the cc-pVQZ has been used. The energy and gradient of the cationic and anionic doublet species has been computed within the unrestricted methodology at the geometry of the neutral systems, while the neutral singlet molecules have been calculated using the restricted formalism.

The numerical differentiation of $\varepsilon_{\text {HOMO }}, \varepsilon_{\text {LUMO }}, \mu_{1}, I$, $A$, and $\mu_{2}$ needed to evaluate Eqs. (16)-(18) has been carried out performing displacements of the equilibrium geometry of $\pm(1,2,4,8,16,32,64) \times 10^{-n}(n=2$ to 5$)$ bohrs. Then, the smallest magnitude displacement that produced a stable derivative has been selected using a Romberg method triangle. $^{24}$

Before starting the evaluation of the $-\sum_{k=1}^{3 N-6} \phi_{k} \Delta Q_{k}$ term in Eq. (34), we have checked for 13 diatomic molecules the consistency of the $\phi_{\alpha}^{+}, \phi_{\alpha}^{-}$, and $\phi_{\alpha}^{0}$ nuclear Fukui functions calculated using the different approximations repre- sented by Eqs. (12) to (14) and (16) to (18). The results obtained are listed in Table I. It is remarkable to see that calculations of the nucleophilic NFF, $\phi_{\alpha}^{+}$, by numerical differentiation of $\left(\delta A / \delta \vec{R}_{\alpha}\right)_{N}$ or $\left(\partial \vec{F}_{\alpha} / \partial N\right)_{\nu}^{+}$yield practically the same values providing reliability on the computed values; while the calculation of $\phi_{\alpha}^{+}$using the orbital approximation $-\left(\delta \varepsilon_{\text {LUMO }} / \delta \vec{R}_{\alpha}\right)_{N}$ results in somewhat different numbers. The same is found for the electrophilic and radical NFFs. It is worth noting that the results obtained by Balawender and Geerlings ${ }^{10}$ for $\phi_{\alpha}^{+}$and $\phi_{\alpha}^{-}$of $\mathrm{H}_{2}, \mathrm{LiH}, \mathrm{BH}, \mathrm{HF}$, $\mathrm{HCl}, \mathrm{N}_{2}, \mathrm{~F}_{2}, \mathrm{CO}, \mathrm{BF}$, and $\mathrm{LiF}$ at the HF/6-31++ $\mathrm{G}^{* *}$ are, in general, in good agreement with those reported in Table I. As already pointed out by Baekelandt ${ }^{8}$ and Geerlings and coworkers, ${ }^{9,10}$ NFF data can be related to the Berlin's function $^{8,13,14}$ to analyze bonding in molecules. Thus, in diatomic molecules a positive nucleophilic NFF is associated to an increasing bond length upon the addition of an electron, which increases the total electron density in antibonding regions. All species studied show positive nucleophilic NFF as indicated by the values obtained from the $\left(\delta A / \delta \vec{R}_{\alpha}\right)_{N}$ and $\left(\partial \vec{F}_{\alpha} / \partial N\right)_{\nu}^{+}$derivatives. Likewise, a negative electrophilic NFF is also related to an increase of the bond length during ionization, which means a reduction of the total electron density in bonding regions upon ionization. We found negative electrophilic NFF for all species studied, except for $\mathrm{BH}, \mathrm{F}_{2}$, $\mathrm{CO}, \mathrm{NF}$, and $\mathrm{BF}$, for which positive electrophilic NFFs are obtained, indicating that the ionization should lead to a decrease of the bond length in these molecules.

Let us now numerically test the validity of Eq. (34). We have checked Eq. (34) for different displacements using the NFF obtained from Eqs. (12) to (14), although we only show in Table II the results for $\Delta Q_{k}=1 \times 10^{-5} \times \sqrt{\mu}$ a.u., where $\mu$ is the reduced mass, because with this displacement the error percentage becomes stable. We note that, from Eq. (8), the first term appearing in Eq. (34) is 
TABLE II. The calculation of the two terms given in Eq. (34) for the three approximations of the electronic and nuclear Fukui functions for 13 diatomic molecules. All values are given in atomic units.

\begin{tabular}{ccccccc}
\hline \hline Molecule & $\int f^{+}(\vec{r}) \Delta \nu(\vec{r}) d \vec{r}$ & $-\phi_{k}^{+} \Delta Q_{k}{ }^{\mathrm{a}}$ & $\int f^{-}(\vec{r}) \Delta \nu(\vec{r}) d \vec{r}$ & $-\phi_{k}^{-} \Delta Q_{k}{ }^{\mathrm{a}}$ & $\int f^{0}(\vec{r}) \Delta \nu(\vec{r}) d \vec{r}$ & $-\phi_{k}^{0} \Delta Q_{k}{ }^{\mathrm{a}}$ \\
\hline $\mathrm{H}_{2}$ & $-5.30 \times 10^{-8}$ & $-5.60 \times 10^{-8}$ & $1.52 \times 10^{-6}$ & $1.52 \times 10^{-6}$ & $7.34 \times 10^{-7}$ & $7.34 \times 10^{-7}$ \\
$\mathrm{LiH}$ & $-1.07 \times 10^{-7}$ & $-9.79 \times 10^{-8}$ & $3.00 \times 10^{-7}$ & $2.79 \times 10^{-7}$ & $9.65 \times 10^{-8}$ & $9.04 \times 10^{-8}$ \\
$\mathrm{BH}$ & $-7.91 \times 10^{-8}$ & $-7.34 \times 10^{-8}$ & $-1.09 \times 10^{-7}$ & $-1.53 \times 10^{-7}$ & $-9.41 \times 10^{-8}$ & $-1.13 \times 10^{-7}$ \\
$\mathrm{NH}$ & $-3.22 \times 10^{-8}$ & $-4.07 \times 10^{-8}$ & $2.80 \times 10^{-7}$ & $2.72 \times 10^{-7}$ & $1.24 \times 10^{-7}$ & $1.15 \times 10^{-7}$ \\
$\mathrm{HF}$ & $-1.01 \times 10^{-7}$ & $-1.30 \times 10^{-7}$ & $8.61 \times 10^{-7}$ & $8.57 \times 10^{-7}$ & $3.80 \times 10^{-7}$ & $3.63 \times 10^{-7}$ \\
$\mathrm{HCl}$ & $-5.20 \times 10^{-8}$ & $-7.97 \times 10^{-8}$ & $2.37 \times 10^{-7}$ & $2.31 \times 10^{-7}$ & $9.25 \times 10^{-8}$ & $7.54 \times 10^{-8}$ \\
$\mathrm{~N}_{2}$ & $-1.79 \times 10^{-8}$ & $-3.28 \times 10^{-8}$ & $3.29 \times 10^{-7}$ & $5.77 \times 10^{-7}$ & $1.55 \times 10^{-7}$ & $2.72 \times 10^{-7}$ \\
$\mathrm{~F}_{2}$ & $-3.44 \times 10^{-6}$ & $-4.75 \times 10^{-6}$ & $-1.21 \times 10^{-6}$ & $-1.36 \times 10^{-6}$ & $-2.32 \times 10^{-6}$ & $-3.05 \times 10^{-6}$ \\
$\mathrm{CO}$ & $-1.90 \times 10^{-7}$ & $-1.67 \times 10^{-7}$ & $-5.95 \times 10^{-7}$ & $-4.42 \times 10^{-7}$ & $-3.92 \times 10^{-7}$ & $-3.05 \times 10^{-7}$ \\
$\mathrm{NF}$ & $-1.15 \times 10^{-6}$ & $-1.32 \times 10^{-6}$ & $-1.26 \times 10^{-6}$ & $-1.46 \times 10^{-6}$ & $-1.20 \times 10^{-6}$ & $-1.39 \times 10^{-6}$ \\
$\mathrm{BF}$ & $-9.55 \times 10^{-8}$ & $-1.14 \times 10^{-7}$ & $-7.36 \times 10^{-7}$ & $-6.90 \times 10^{-7}$ & $-4.16 \times 10^{-7}$ & $-4.02 \times 10^{-7}$ \\
$\mathrm{NO}{ }^{+}$ & $-3.10 \times 10^{-6}$ & $-3.26 \times 10^{-6}$ & $1.05 \times 10^{-6}$ & $1.36 \times 10^{-6}$ & $-1.02 \times 10^{-6}$ & $-9.48 \times 10^{-7}$ \\
$\mathrm{LiF}$ & $-1.85 \times 10^{-7}$ & $-1.60 \times 10^{-7}$ & $6.53 \times 10^{-7}$ & $5.89 \times 10^{-7}$ & $2.34 \times 10^{-7}$ & $2.15 \times 10^{-7}$ \\
\hline
\end{tabular}

${ }^{a}$ The values of nuclear Fukui functions are obtained using Eqs. (12), (13), and (14). The $\Delta Q_{k}$ employed in all these cases is $1 \times 10^{-5} \times \sqrt{\mu}$ a.u., where $\mu$ is the reduced mass.

$$
\begin{aligned}
& \int f^{+}(\vec{r}) \Delta \nu(\vec{r}) d \vec{r} \\
& \cong \int\left(\rho_{N+1}^{0}(\vec{r})-\rho_{N}^{0}(\vec{r})\right)\left(\nu(\vec{r})-\nu^{0}(\vec{r})\right) d \vec{r} \\
& =\int \rho_{N+1}^{0}(\vec{r}) \nu(\vec{r}) d \vec{r}-\int \rho_{N+1}^{0}(\vec{r}) \nu^{0}(\vec{r}) d \vec{r} \\
& \quad-\int \rho_{N}^{0}(\vec{r}) \nu(\vec{r}) d \vec{r}+\int \rho_{N}^{0}(\vec{r}) \nu^{0}(\vec{r}) d \vec{r},
\end{aligned}
$$

where $\rho_{N}^{0}(\vec{r})$ and $\rho_{N+1}^{0}(\vec{r})$ are the electronic density at the equilibrium geometry for a system with $N$ and $N+1$ electrons, respectively, while $\nu^{0}(\vec{r})$ is the external potential at the equilibrium geometry and $\nu(\vec{r})$ is the external potential obtained increasing or decreasing the equilibrium bond length. The $\int \rho_{N+1}^{0}(\vec{r}) \nu^{0}(\vec{r}) d \vec{r}$ and $\int \rho_{N}^{0}(\vec{r}) \nu^{0}(\vec{r}) d \vec{r}$ integrals are explicitly the electron-nucleus potential energy of the system with $N+1$ and $N$ electrons at the equilibrium geometry, respectively. The $\int \rho_{N+1}^{0}(\vec{r}) \nu(\vec{r}) d \vec{r}$ and $\int \rho_{N}^{0}(\vec{r}) \nu(\vec{r}) d \vec{r}$ integrals in Eq. (38) are the electron-nucleus potential energy introducing the modified external potential $\nu(\vec{r})$, but using the $N+1$ and $N$ electronic density at the equilibrium geometry. All integrals present in Eq. (38) have been obtained from electron-nucleus potential energies computed with the GAUSSIAN 98 program. ${ }^{21,25}$ Equivalent expressions to Eq. (38) can be used to evaluate the integrals containing $f^{-}(\vec{r})$ and $f^{0}(\vec{r})$.

As can be seen in Table II, the difference between the first and the second term of Eq. (34) is usually quite small for all systems studied and for the different nucleophilic, electrophilic, and radical approximations to the electronic and nuclear Fukui functions. The similarity between the values of the NFF computed using the two most reliable approximations make us confident about the accuracy of the calculated NFFs, while operational equations that provide the electronic Fukui function [Eqs. (8) to (10)] are unavoidably approximate. Thus, in our opinion, the numerical errors that produce the differences observed may be larger in the computation of the integrals of the type $\int f(\vec{r}) \Delta \nu(\vec{r}) d \vec{r}$ than in the evaluation of the $-\sum_{k=1}^{3 N-6} \phi_{k} \Delta Q_{k}$ term. Figure 1 depicts the good correlation observed between the $\int f^{-}(\vec{r}) \Delta \nu(\vec{r}) d \vec{r}$ and the $-\sum_{k=1}^{3 N-6} \phi_{k}^{-} \Delta Q_{k}$ values giving an equation with a slope very

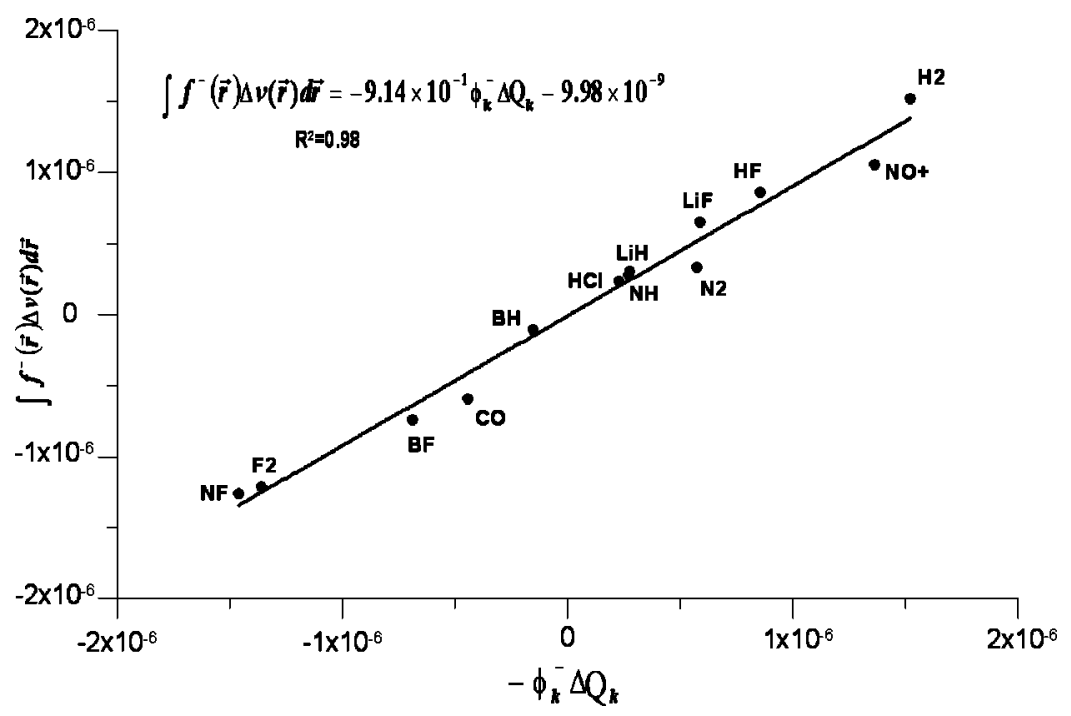

FIG. 1. A representation of the linear equation $\int f^{-}(\vec{r}) \Delta \nu(\vec{r}) d \vec{r}=-\phi_{k}^{-} \Delta Q_{k}$ for the 13 molecules studied. 
TABLE III. The calculation of the (1/2) $\iint \chi\left(\vec{r}, \vec{r}^{\prime}\right) \Delta \nu(\vec{r}) \Delta \nu\left(\vec{r}^{\prime}\right) d \vec{r} d \vec{r}^{\prime}$ integral from the evaluation of the remaining two terms in Eq. (36) for the 13 diatomic molecules studied. All values are given in atomic units. ${ }^{\mathrm{a}}$

\begin{tabular}{cccc}
\hline \hline Molecule & $\frac{1}{2} \lambda_{k} \Delta Q_{k}^{2}$ & $\frac{1}{2}\left(\frac{\partial^{2} V_{n n}}{\partial Q_{k}^{2}}\right)_{N} \Delta Q_{k}^{2}$ & $\frac{1}{2} \iint \chi\left(\vec{r}, \vec{r}^{\prime}\right) \Delta \nu(\vec{r}) \Delta \nu\left(\vec{r}^{\prime}\right) d \vec{r} d \vec{r}^{\prime}$ \\
\hline $\mathrm{H}_{2}$ & $2.00 \times 10^{-11}$ & $3.75 \times 10^{-11}$ & $-1.75 \times 10^{-11}$ \\
$\mathrm{LiH}$ & $3.40 \times 10^{-12}$ & $1.07 \times 10^{-11}$ & $-7.34 \times 10^{-12}$ \\
$\mathrm{BH}$ & $1.08 \times 10^{-11}$ & $4.08 \times 10^{-11}$ & $-3.00 \times 10^{-11}$ \\
$\mathrm{NH}$ & $2.30 \times 10^{-11}$ & $9.89 \times 10^{-11}$ & $-7.60 \times 10^{-11}$ \\
$\mathrm{HF}$ & $3.62 \times 10^{-11}$ & $1.85 \times 10^{-10}$ & $-1.48 \times 10^{-10}$ \\
$\mathrm{HCl}$ & $1.83 \times 10^{-11}$ & $1.24 \times 10^{-10}$ & $-1.06 \times 10^{-10}$ \\
$\mathrm{~N}_{2}$ & $1.08 \times 10^{-10}$ & $6.00 \times 10^{-10}$ & $-4.92 \times 10^{-10}$ \\
$\mathrm{~F}_{2}$ & $2.88 \times 10^{-11}$ & $5.14 \times 10^{-10}$ & $-4.85 \times 10^{-10}$ \\
$\mathrm{CO}$ & $7.64 \times 10^{-11}$ & $5.31 \times 10^{-10}$ & $-4.55 \times 10^{-10}$ \\
$\mathrm{NF}$ & $2.72 \times 10^{-11}$ & $4.59 \times 10^{-10}$ & $-4.32 \times 10^{-10}$ \\
$\mathrm{BF}$ & $2.95 \times 10^{-11}$ & $3.45 \times 10^{-10}$ & $-3.16 \times 10^{-10}$ \\
$\mathrm{NO}{ }^{+}$ & $1.14 \times 10^{-10}$ & $7.70 \times 10^{-10}$ & $-6.56 \times 10^{-10}$ \\
$\mathrm{LiF}$ & $8.61 \times 10^{-12}$ & $1.07 \times 10^{-10}$ & $-9.79 \times 10^{-11}$ \\
\hline \hline
\end{tabular}

a The $\Delta Q_{k}$ employed in all these cases is $1 \times 10^{-5} \times \sqrt{\mu}$ a.u., where $\mu$ is the reduced mass.

close to 1, which is the expected value according to Eq. (34). Similar results are obtained for the analogous relations involving the nucleophilic and radical Fukui functions. We have checked Eq. (34) using different basis sets and we have found that, in general, the correlation between the $\int f(\vec{r}) \Delta \nu(\vec{r}) d \vec{r}$ and the $-\sum_{k=1}^{3 N-6} \phi_{k} \Delta Q_{k}$ values improves when the quality of the basis set used increases. Finally, we have also tested Eq. (34) using the B3LYP method and we have found similar results to those given in Table II.

As a final point, Table III gathers the computed values for the $(1 / 2) \sum_{k=1}^{3 N-6} \lambda_{k} \Delta Q_{k}^{2}$ and $(1 / 2) \sum_{k=1}^{3 N-6} \sum_{l=1}^{3 N-6}\left(\partial^{2} V_{n n} /\right.$ $\left.\partial Q_{k} \partial Q_{l}\right)_{N} \Delta Q_{k} \Delta Q_{l}$ terms appearing in Eq. (36). The integral $(1 / 2) \iint \chi\left(\vec{r}, \vec{r}^{\prime}\right) \Delta \nu(\vec{r}) \Delta \nu\left(\vec{r}^{\prime}\right) d \vec{r} d \vec{r}^{\prime}$ is evaluated as the difference between the two previous calculated terms, according to Eq. (36). It is also worthwhile noting that in line with Eq. (37), the second term corresponds to the integral $(1 / 2) \iint X\left(\vec{r}, \vec{r}^{\prime}\right) \Delta \nu(\vec{r}) \Delta \nu\left(\vec{r}^{\prime}\right) d \vec{r} d \vec{r}^{\prime}$. From Eq. (32), one can see that, starting from a system in its equilibrium geometry, the change (up to second order) in the energy of the molecular system due to a molecular distortion can be split into an electronic and a nuclear term. The electronic term, $(1 / 2) \iint \chi\left(\vec{r}, \vec{r}^{\prime}\right) \Delta \nu(\vec{r}) \Delta \nu\left(\vec{r}^{\prime}\right) d \vec{r} d \vec{r}^{\prime}$, is the change in the electronic energy due a change in the external potential, while the nuclear part, (1/2) $\iint X\left(\vec{r}, \vec{r}^{\prime}\right) \Delta \nu(\vec{r}) \Delta \nu\left(\vec{r}^{\prime}\right) d \vec{r} d \vec{r}^{\prime}$, gives the change in nuclear repulsion energy due to the same perturbation. Interestingly, for the diatomic molecules studied, the change in electronic energy due to molecular distortion is always negative and smaller in absolute value than the nuclear energy variation, which is always positive (see Table III). It would be interesting to investigate whether the sign of these integrals are preserved in polyatomic molecules. Remarkably, those molecules that suffer a large nuclear energetic change in the distortion (for instance, the $\mathrm{N}_{2}, \mathrm{~F}_{2}, \mathrm{CO}$ and $\mathrm{NO}^{+}$species), also experience a large electronic energetic change. Let us finish by mentioning that the values of $(1 / 2) \iint \chi\left(\vec{r}, \vec{r}^{\prime}\right) \Delta \nu(\vec{r}) \Delta \nu\left(\vec{r}^{\prime}\right) d \vec{r} d \vec{r}^{\prime}$ in Table III can be a good starting point for discussing the validity of possible analytical expressions for the electronic linear response function.

\section{CONCLUSIONS}

In this paper, we have derived a set of connections among several nuclear and electronic indexes of reactivity in the framework of the conceptual Density Functional Theory by using an expansion of the energy functional in terms of the total number of electrons and the normal coordinates within a canonical ensemble. The relations obtained provide explicit links between important quantities related to the chemical reactivity of a system.

First, we have demonstrated that the derivative of the electronic energy with respect to the external potential of a system in its equilibrium geometry is equal to the negative of the nuclear repulsion derivative with respect to the external potential, giving a proof up to the first order for the so-called "Quantum Chemical le Chatelier Principle." Second, following this particular approach, we have given an alternative proof the relation between the nuclear and the electronic Fukui functions, and, for the first time, we have numerically checked this relation. Finally, the nuclear linear response function has been introduced and a relation of this function with the electronic linear response function and the force constant has been provided.

\section{ACKNOWLEDGMENTS}

Support for this work under Grant No. BQU2002-0412C02-02 from the Dirección General de Enseñanza Superior e Investigación Científica y Técnica (MEC-Spain), from the Departament d'Universitats, Recerca i Societat de la Informació (DURSI) of the Generalitat de Catalunya through an AIRE Grant, and from the Chilean CONYCIT is acknowledged. M.T. thanks the Generalitat de Catalunya for financial help through CIRIT Project No. FI/01-00699. M.S. is indebted to the DURSI for financial support through the Distinguished University Research Promotion 2001.

${ }^{1}$ R. G. Parr and W. Yang, Density-Functional Theory of Atoms and Molecules (Oxford University Press, New York, 1989).

${ }^{2}$ H. Chermette, J. Comput. Chem. 20, 129 (1999).

${ }^{3}$ R. G. Parr and R. G. Pearson, J. Am. Chem. Soc. 105, 7512 (1983).

${ }^{4}$ M. Berkowitz, S. K. Ghosh, and R. G. Parr, J. Am. Chem. Soc. 107, 6811 (1985).

${ }^{5}$ R. G. Parr and W. Yang, J. Am. Chem. Soc. 106, 4049 (1984).

${ }^{6}$ P. Senet, J. Chem. Phys. 107, 2516 (1997).

${ }^{7}$ M. H. Cohen, M. V. Ganduglia-Pirovano, and J. Kudrnovský, J. Chem. Phys. 101, 8988 (1994); M. H. Cohen, M. V. Ganduglia-Pirovano, and J. Kudrnovský, ibid. 103, 3543 (1995).

${ }^{8}$ B. G. Baekelandt, J. Chem. Phys. 105, 4664 (1996).

${ }^{9}$ F. De Proft, S. Liu, and P. Geerlings, J. Chem. Phys. 108, 7549 (1998).

${ }^{10}$ R. Balawender and P. Geerlings, J. Chem. Phys. 114, 682 (2001).

${ }^{11}$ T. Koopmans, Physica (Amsterdam) 1, 104 (1934).

${ }^{12}$ R. Balawender and L. Komorowski, J. Chem. Phys. 109, 5203 (1998).

${ }^{13}$ T. Berlin, J. Chem. Phys. 19, 208 (1951).

${ }^{14}$ R. Balawender, F. De Proft, and P. Geerlings, J. Chem. Phys. 114, 4441 (2001).

${ }^{15}$ B. G. Baekelandt, A. Cedillo, and R. G. Parr, J. Chem. Phys. 103, 8548 (1995).

${ }^{16}$ P. Ordon and L. Komorowski, Chem. Phys. Lett. 292, 22 (1998).

${ }^{17}$ E. Chamorro, P. Fuentealba, and R. Contreras, J. Chem. Phys. 115, 6822 (2001). 
${ }^{18}$ P. W. Ayers and R. G. Parr, J. Am. Chem. Soc. 122, 2010 (2000); P. W. Ayers and R. G. Parr, ibid. 123, 2007 (2001).

${ }^{19}$ P. Geerlings, F. De Proft, and W. Langenaeker, Chem. Rev. 103, 1793 (2003).

${ }^{20}$ P. G. Mezey, Int. J. Quantum Chem. 25, 853 (1984); P. G. Mezey, Chem. Phys. Lett. 47, 70 (1977).

${ }^{21}$ M. J. Frisch, G. W. Trucks, H. B. Schlegel et al., GAUSSIAN 98, Revision A.11, Gaussian, Inc., Pittsburgh, PA, 1998.

${ }^{22}$ C. C. J. Roothaan, Rev. Mod. Phys. 35, 69 (1951).
${ }^{23}$ T. H. Dunning, Jr., J. Chem. Phys. 90, 1007 (1989); D. E. Woon and T. H. Dunning, Jr., ibid. 100, 2975 (1994); R. A. Kendall, T. H. Dunning, Jr., and R. J. Harrison, ibid. 96, 6796 (1992).

${ }^{24}$ P. J. Davis and P. Rabinowitz, Numerical Integration (Blaisdell, London, 1967), p. 166.

${ }^{25}$ The $\int \rho_{N+1}^{0}(\vec{r}) \nu(\vec{r}) d \vec{r}$ and $\int \rho_{N}^{0}(\vec{r}) \nu(\vec{r}) d \vec{r}$ integrals can be evaluated analytically with the GAUSSIAN 98 program using the keywords "massage" and "guess= read" to freeze the density at that of the equilibrium geometry and adding punctual charges to move nuclei to the new positions. 